\title{
Frontières
}

\section{Pratiques rituelles du temps du mourir et formes actuelles de la belle mort}

\section{Luce Des Aulniers}

Volume 20, numéro 1, automne 2007

La « bonne mort»

URI : https://id.erudit.org/iderudit/017943ar

DOI : https://doi.org/10.7202/017943ar

Aller au sommaire du numéro

\section{Éditeur(s)}

Université du Québec à Montréal

ISSN

1180-3479 (imprimé)

1916-0976 (numérique)

Découvrir la revue

Citer cet article

Des Aulniers, L. (2007). Pratiques rituelles du temps du mourir et formes actuelles de la belle mort. Frontières, 20(1), 22-26.

https://doi.org/10.7202/017943ar
Résumé de l'article

Cet article examine comment le rite contribue à une anthropologie du temps, en particulier le temps long et composite du rapport à la mort. Le rite apparaît ainsi comme un creuset et un véhicule de mise en liens ou de symbolique. La marche est proposée comme un exemple d'une activité quotidienne qui peut devenir pour un grand malade un rituel de partage de sens avec autrui en préparation à la mort. Trois nouvelles représentations de la mort sont aussi discutées : la mort comme résolution d'un problème avec la vie, comme rejet du corps et comme déni de la violence. Le passage de la bonne à la belle mort rend visible un rapport au temps : on passerait ainsi de la bonne mort, celle qui vient à son heure, à la belle mort, qui arrive lorsque la personne qui se meurt accepte de l'inviter. 


\section{Résumé}

Cet article examine comment le rite contribue à une anthropologie du temps, en particulier le temps long et composite du rapport à la mort. Le rite apparaît ainsi comme un creuset et un véhicule de mise en liens ou de symbolique. La marche est proposée comme un exemple d'une activité quotidienne qui peut devenir pour un grand malade un rituel de partage de sens avec autrui en préparation à la mort. Trois nouvelles représentations de la mort sont aussi discutées: la mort comme résolution d'un problème avec la vie, comme rejet du corps et comme déni de la violence. Le passage de la bonne à la belle mort rend visible un rapport au temps: on passerait ainsi de la bonne mort, celle qui vient à son heure, à la belle mort, qui arrive lorsque la personne qui se meurt accepte de l'inviter.

Mots clés: rite - bonne mort anthropologie du temps.

\begin{abstract}
This article examines how rite contributes to an anthropology of time, especially time considered as long and composite in it's relation with death. The rite appears as a crucible and as a vehicle for establishing connections or symbolism. Walking is suggested as an example of an everyday activity that may become for a seriously ill person a ritual of shared meaning with others in preparation for death. Three new representations of death are also discussed: death as solving a problem with life, as a rejection of the body and as a denial of violence. The transition from the good to the fine death reveals a relationship with time: we would therefore move away from a good death, one that is timely, towards a fine death, which happens when the dying person agrees to welcome it.
\end{abstract}

Keywords: rite - good death anthropology of time.

$\begin{array}{llllllll}\text { A } & R & \text { T } & \text { I } & \text { C } & \text { L } & \text { E } & \text { S }\end{array}$

\section{Pratiques rituelles du temps du mourir et formes actuelles de la belle mort ${ }^{1}$}

Luce Des Aulniers, Ph. D., anthropologue, professeure titulaire,

Département de communication sociale et publique, Études interdisciplinaires sur la mort, UQAM.

Temps du mourir, certes, mais... de quelle avant-mort parlons nous?

La citation ci-après présente à cet égard un double mérite qui se donne d'autant comme objectifs de ce texte. En premier lieu, il s'agira d'extirper un enjeu inhérent à la concentration si ce n'est à la crispation existentielle, professionnelle - et même épistémologique - sur l'avantmort proche, dite "phase terminale». Ensuite, cet extrait posant la pierre d'angle de la présente contribution, à savoir une anthropologie du temps, nous pourrons envisager comment le rite contribue à cette anthropologie du temps, voire viendrait subvertir les conventions contemporaines relatives à ce que nous nous représentons comme une belle mort, qui alors, ne serait pas que concernée par le temps proche du mourir.

Cet extrait de Noces de sable évoque Gabriel au chevet de son père Victor:

Gabriel garde les yeux fermés, car, lui, n'a jamais su prier autrement que pour voir s'allumer le visage de sa mère lorsqu'il était petit. Victor aime entendre la prière si parfaite, si fervente, le chant à la fois solitaire et fraternel de ce garçon qui est son fils. Un tel aveu de désœuvrement le rassure, lui rappelle qu'ici-bas, on n'est pas moins vulnérable ni plus heureux que ces autres qui attendent, qu'en tout cas vivants et morts ne réclament qu'une seule et même chose, d'être consolés. Mais de quoi, se demande alors Gabriel, de quoi les vivants veulent-ils tant obtenir consolation? Peut-être de la faiblesse dont ils ont fait preuve, un jour, devenus adultes, quand ils ont abdiqué, quand ils ont baissé les bras et constaté leur peu de confiance et d'amour envers le monde qui leur était offert en héritage. Peut-être, se dit Gabriel, en pensant à son propre père, voulons-nous être consolés, nous les vivants, de ces erreurs que nous commettons, de mille petites failles quotidiennes, consolés surtout d'avoir dû nous soumettre à une loi terrible qui nous rassemble et nous aveugle, nous fait accepter les manquements de cette sorte et nous fait croire à leur banalité.

[...]

Pourquoi, se demande Gabriel, en sentant la main de Victor sur son épaule, attendons-nous que les forts soient devenus faibles pour les toucher et tenter d'atteindre le centre de leur être? Avons-nous si peur de l'existence qu'il faille ne s'approcher des autres que lorsqu'ils ne vivent qu'à demi, sommes-nous assez mesquins pour attendre cela - la mort prenant possession de la maison -, pour enfin être à l'aise avec les nôtres et venir exiger qu'ils nous apprennent encore quelque chose, pour leur voler ce reste 
de force et de chaleur dont ils ont tant besoin? Dieu, pardonne-nous, avons-nous donc été si petits, si peu vivants? (Leclerc, 2000, p. 32)

\section{LE TEMPS LONG ET COMPOSITE DU RAPPORT À LA MORT}

Alors, paradoxalement, se pourrait-il que nous sachions bien peu du mourir quand nous le cantonnons aux derniers jours? Se peut-il que le «bien» comme le «mal» mourir - et ce qui se tient entre les deux - s'entendent aussi autrement? En effet, la manière de mourir se profilerait dès à partir du moment où, par sa maladie, une personne s'avère consciente de la réalité plus dramatiquement en acte en elle. D'où le caractère fondamental de l'information, espérée, retenue, reçue, demandée, partagée.

Bien plus, les derniers moments de la vie seraient largement déterminés par l'élaboration permise dans la traversée littéralement - de la maladie grave.

Enfin, si on mire cette traversée encore plus en amont, on y voit se condenser un rapport à la mort travaillé sur toute la vie, consciemment, inconsciemment, préconsciemment.

Autrement dit et par analogie, on sait que ce qui importe de tout voyage n'est pas que l'arrivée, le terminus, la résolution de la tension plus ou moins délicieuse ${ }^{2}$ qui lui est impartie. Ce sont aussi l'itinéraire, les escales, les rencontres, l'imprévu, la cohérence profonde avec son être, au travers des nécessaires contradictions, toutes choses qui altèrent, embrument ou font scintiller le regard au terme du périple. Ainsi, au bout de cet itinéraire vitalisé, la question de bien ou mal mourir ne se pose pas tel un horizon, encore moins comme une marque sociale d'efficience ou d'assurance du bon souvenir des survivants. Bien ou mal ou ce qui bouge entre les deux - comme un enfant - se donne et se dépose de surcroît. Un peu par boutade, je proposerais que la bonne ou la belle mort - j'y reviendrai - s'avère sans qu'on l'appelle à toute force, en se décentrant de l'obsession contemporaine de la date butoir, de l'objectif managérial; elle arrive, curieusement dans une sorte de congruence non clamée... et elle signe le sens véritable de l'holisme qui, outre ce que nous en savons tous, consiste justement à penser l'être - comme les cultures - dans ce temps long.

Or, a priori, ce temps long nous échappe quand nous nous identifions uniquement comme intervenants en santé, puisqu'il se déploie en bonne part hors de notre vue, dans des lieux investis par ces grands malades. Mais ce n'est pas une raison pour ignorer ce qui se passe.
Passe, passage, j'arrive bientôt au rite.

\section{UN PASSAGE SIGNÉ DE} L'AUTONOMIE ET DE LA LIBERTÉ

"Votre mot de passe?» pourraient métaphoriquement réclamer aux grands malades les passeurs-accompagnants; les uns et les autres seraient à leur insu ou à leur corps défendant entraînés dans les obligations technocratiques de notre monde qui dicte de manière plus ou moins subtile les rythmes de la vie quotidienne et les formes de pensée.

Toutefois, beaucoup de nos contemporains, à la fois actifs et songeurs sur le chemin de leur mort, souhaitent affronter l'angoisse sans trop d'intermédiaires, à telle enseigne qu'on ne trouve pas autour d'eux de définisseur de la mort convenue, pas de dépositaire de savoir, ni de grand prêtre ou prêtresse de l'ultime performance, ou de commentateur-voyeur de la limite, et non plus, d'auxiliaire du politique. En effet, éventuelle nouveauté contemporaine, la volonté - inconsciente - de contrôle émanant des édicteurs et des exhortateurs de tout acabit serait repérée, puis parfois boutée par les premiers concernés par la mort qui «arrive». Ou en tout cas, mise en question. Et très souvent évitée... (Pointe déjà cette inquiétude : à l'extrême, les vœux de mort de nos patients viseraient-ils notamment à échapper à une sollicitude, sans doute trop amène ou pas assez autoréflexive...? Joli paradoxe!) Bref, l'histoire millénaire des modèles et modéliseurs vacillerait-elle un brin ?... Alors ici, tout au plus, rencontre-t-on des distillateurs ou distillatrices de maternage, des ouvreurs de questions du simple sourire dans le regard, des facilitateurs discrets de mise en place des conditions du rite: or, ces conditions tiennent essentiellement dans «l'enbonheureusement» (comme le disait un patient) des cinq sens, dans l'harmonisation fluide de l'univers significatif, histoire de se mettre au pas de «l'appétence relationnelle» du malade et du mourant dont parlait M. de M'Uzan (1977), appétence toutefois complexe dans ses résonances et dont je ne peux traiter ici.

\section{UN RITE CREUSET ET VÉHICULE DE MISE EN LIENS OU DE SYMBOLIQUE}

Quoi qu'il en soit, le rite dont je parle est issu des «profanes». Et répétons que face à la mort... eh bien, nous le sommes tous.

Comment donc repère-t-on le dit rite?

Louis-Vincent Thomas, comme à propos de tant d'angles sur l'humanité s'instituant dans la mort, nous mettait sur la piste: "Chaque fois que la signification d'un acte réside dans sa valeur symbolique plus que dans sa finalité mécanique, nous sommes déjà sur la voie d'une conduite rituelle»(Thomas, 1991, p. 115). Cette remarque $\mathrm{du}$ grand socioanthropologue place d'emblée le rite dans une position relativement contreculturelle, dans la mesure où la sensibilité contemporaine en serait venue à éprouver quelque difficulté à concevoir une situation autre que dans ses recours «automatiques", dit-on, à la technologie, et ce, nonobstant les bénéfices de cette dernière au plan biomédicohospitalier; on pense ici d'évidence à la biotechnologie, à ses appareillages aussi bien qu'à la pharmacologie, avec certaines expressions-limites comme celle de "pain killer» (sic: by the way, what are we killing ?); on pense aussi à ce que l'on peut aisément alimenter quand on professionnalise un champ de pratique, à savoir la technologie relationnelle: les bons mots au bon moment, pouvant à l'extrême fantastiquement garantir une bonne mort. Ces deux logiques techno procèdent de savoirs durs, catégoriels, phasiques, prédictifs. Pour ne pas parler des enjeux quantitatifs en termes économiques, de pouvoir ou de prestige, cachés sous l'utilitarisme.

Mais surtout, ces technologies garantes du salut temporel nous leurrent lorsqu'elles prétendent que le génie humain s'y est pleinement exprimé. C'est que mine de rien, elles se sont tour à tour autonomisées, en ce sens qu'elles peuvent à la limite rouler d'elles-mêmes. Pis, il arrive qu'elles ne tiennent plus compte de la personnesujet qu'elles devaient à l'origine soulager ou soutenir. Si par exemple, et encore, dans certains espaces privilégiés de pays riches, la recherche sur la douleur, ou sur les comportements des malades, imbibe désormais le paysage de l'avant-mort - et c'est tant mieux! -, nous ne saurions laisser les technologies le saturer, s'aveuglant dès lors aux traverses de sens imprévisibles, mercurielles, que fait découvrir la maladie grave. On peut par conséquent se demander si celles et ceux qui mettent en place des rites, tout simples, leur permettant de mettre au jour leur vie dans l'avant-mort, ne le feraient pas en guise de subversion, justement de la logique technologisante, par exemple en palliant l'incertitude que cette dernière n'ose pas affronter. Mais peut-être sont-ils ailleurs. Un peu à la manière des poètes, autopoeïn. Autocréateurs.

Qu'est-ce donc que ce rite? "Un ensemble d'actes et de signes matériels à haute teneur symbolique, marquant l'expérience d'un événement et d'une transformation perçus comme mystérieux - si ce n'est menaçants pour la vie - et appelant le dépassement» (Des Aulniers, 1997, p. 554). 
On le constate, on s'éloigne de la mesure de la technologie, qui n'a rien à voir avec le sens de la mesure.

Aussi plusieurs activités quotidiennes peuvent-elles être décodées comme étant rituelles, parsemées de symboles, incandescents autant qu'inconscients des archétypes de l'humanité alors à l'œuvre, aiguillonnés du fait de se sentir à l'orée de l'ultime limite, de l'ultime manque...

\section{MARCHER DEVANT LA MORT}

Prenons l'exemple de la marche, activité connue depuis trois millions sept cent mille ans et, sous sa forme de mobilisation collective, justement reconnue aujourd'hui comme une riposte à l'envahissement technologique et ses avatars, notamment nucléaires (voir notamment Solnit, 2002).

Suivons l'itinéraire du malade qui fera pivoter ses pas et l'imaginaire induit de résistance à la maladie en "préparation» à la mort.

La marche rend compte de la conscience du temps, de la présence de la menace, singulièrement quand le marcheur commente son rythme. Ou il n'a pas besoin: on n'a qu'à observer ce qu'il regarde, la manière, le visage, le corps, ce qu'il désigne aussi, pour peu que l'on emboîte son pas. Il nous montre du précaire et puis le cyclique, ou encore le mouvement de l'eau, en les saluant... Au détour, il loue l'intelligence de l'aménagement ou déplore la bêtise des exploitants. Il touche du patrimoine collectif. Puis il nous entraîne tout à coup dans un sentier de lui inconnu. Tiens donc, l'inconnu ouvre sur de l'ampleur? Il tape le sol du pied ou de la canne, sondant l'identité terrienne... De toutes les manières, il ressent comment la marche transcende son existence individuelle. Il y plonge d'autant; il peut à la fois sauvegarder ce qui reste de forces et évoquer son enfance, ses vieux, partis si loin rejoindre des dieux qu'il aime à croire bienfaisants. Il relatera aussi une histoire de soins qui ramène à l'élément fondateur de toute existence et dans le cas de soin sécure, fondateur précoce du sentiment d'être; de but en blanc, apparemment, il fera défiler ses soignants et, au premier plan, ceux qui métissent haute technicité et chaleur d'âme.

Parfois il marche seul, parfois en cadence avec d'autres, certains autres ayant été douloureusement abandonnés à cause de leur réflexe de rentabilité relationnelle. Il déambule avec ceux patients, clairvoyants; pour ces derniers, il quitte le sentier et va cueillir quelque fleur, petit fruit ou champignon. Se ressoudent les connivences, se déploient le don et le contre-don. Mine de rien, marcher canalise ce qu'il perçoit comme puissances internes

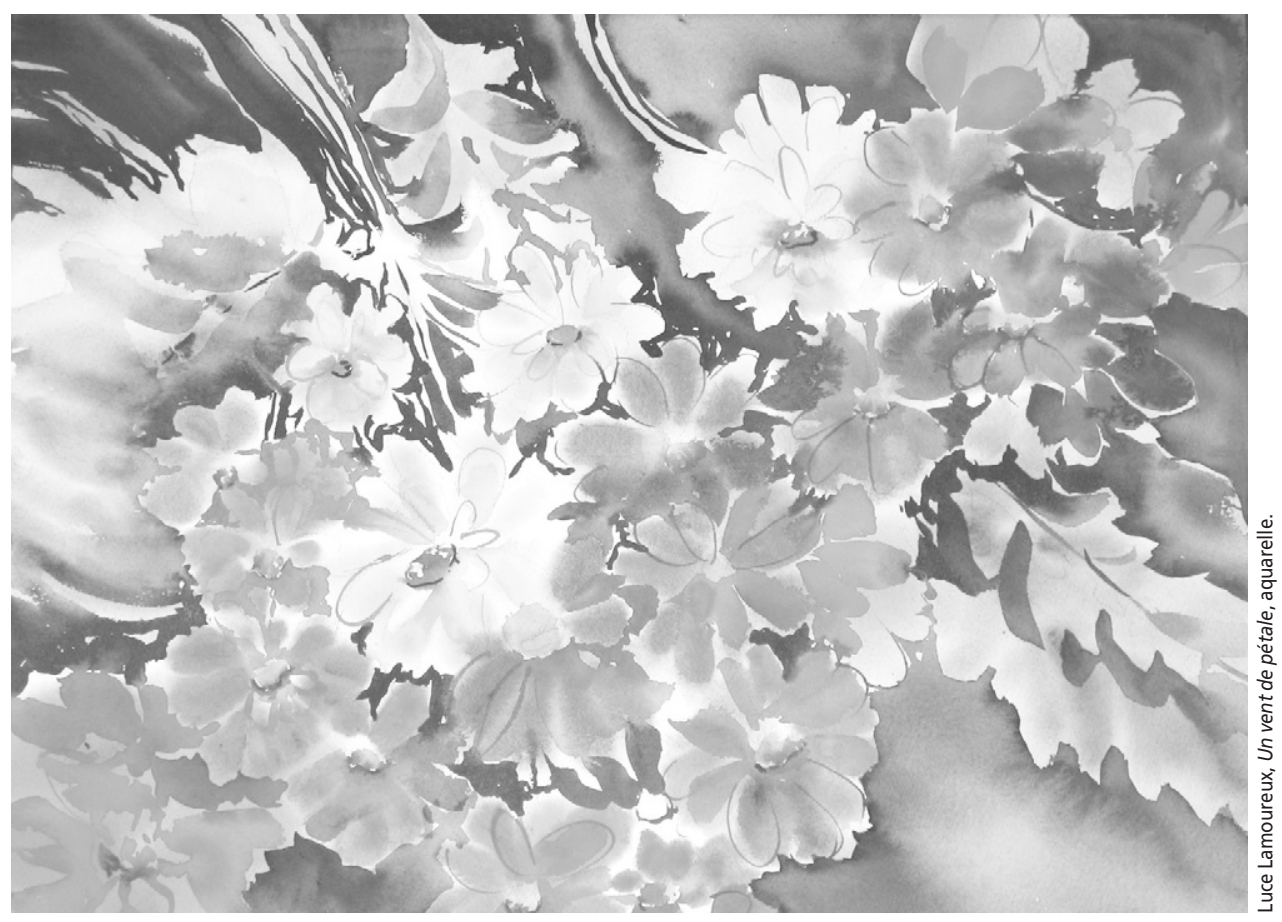

déséquilibrantes et, de ce fait, doublement angoissantes. C'est qu'en marchant, il ne tient plus tant que ça à tromper la peur, il se fond dans le paysage et $\mathrm{y}$ abandonne le désordre de sa maladie. "Ça me repose», qu'il dit. Préfiguration de l'ultime repos. Mais la transcendance de la souffrance et de l'angoisse ne se limite pas au paysage.

Revenu à la maison, trier ses objetssouvenirs s'effectue calmement, résolument: il joue mieux avec ses petits-enfants, écoute les ados dans leurs tourments, discute des événements du monde. Il se force à se concentrer pour lire. Parfois, il se rend au cimetière, il en aime le silence, la pourriture rebutante est moins obsédante; cela le violente, mais il sait qu'il y a des violences incontournables. En revanche, il aide à décorer le sapin de Noël. Il se rappelle des histoires égarées dans sa mémoire et tout le monde rigole. Il s'inscrit dans du cyclique et dans sa mythologie personnelle. Il clarifie son rapport au monde, ça l'aide, il répète: «Y a pas mal de brume, hein? » Vous lui demandez s'il entend la corne de brume. Alors la pensée magique ne se consacre pas au jeu $\mathrm{du}$ «comme si » la mort n'existait pas; elle parie plutôt que cette mort ne s'emparera pas de toute la maison... Bref, que, avant la mort et après, saluant justement cette mort, l'espérance réside dans la foi en une quelconque immortalité.

Vous aviez marché avec cet homme, vous le lui aviez proposé, lorsqu'il s'ennuyait. Hospitalisé, vous êtes tranquillement allé vers lui pour la nuit à préparer : il a esquissé un sourire. Le lendemain, il se soucie de son apparence, et fait quelques pas : il prend contrepied de la laideur de la maladie et de l'immobilisme, symboles universels de mort. Quand il a eu cette crise de douleur, vous lui avez demandé ce qui montait et alors il a hoqueté de ce qu'il ne pouvait plus arpenter l'espace, seulement le temps. Après sa sieste, il vous a fait le récit d'une excursion en canot. À raison, vous vous dites qu'il est bien lucide sur son sort. De temps en temps, à l'improviste, revenant de sa tournée en chaise roulante, il pointe ce qui cloche sur votre aile de soins. Il ne vous parle pas de sa mort, mais de ce qui tue le goût de vivre. Et de ce qui l'a fait vivre. Et encore. Ce disant, il tremble un peu.

Maintenant, il est grabataire; il rêve éveillé devant l'affiche d'un sous-bois qu'il odore. Comme s'il y était. Puis, c'est couru, il en vient à ne presque plus bouger; il envie les chaussures de marche que sa vieille amie n'a pas eu le temps d'enlever avant la visite. Elle les ôte, les place sous le lit. Et c'est reparti, le récit. Cette fois, il l'agrémente de sa vision de la communauté des morts. Le dimanche qui suit, son petit-fils de 4 ans arrive avec ses voitures miniatures et il lui demande de les rouler avec lui sur des plis de draps; le papy acquiesce, puis trop fatigué, lui souffle: «Tu sais, je préfère marcher. » Une semaine plus tard, l'enfant revient, même requête. Le papy, dans les sueurs froides de l'agonie, ne lui répond pas, alors l'enfant déclare: "Bon bien, si tu veux pas, je vais les faire marcher tout seul. » Les heures s'égrènent. Les enfants de ce père, enfin, deux d'entre eux qui le peuvent, sans se concerter, murmurent pour lui cette mélodie: «Un Canadien errant /Banni de ses foyers / Parcourait en pleurant /Des pays 
étrangers. /Si tu vois mon pays / Mon pays malheureux/ bis/Va dire à mes amis /Que je me souviens d'eux...»

Il s'assoupit, on n'entend plus son souffle. Ses pantoufles sont restées sous son lit, à sa demande.

\section{LE RITE DISCRET ET RÉPARATEUR}

Le rite de passage consiste à filer les continuités, avec tant d'aisance que les discontinuités sont accueillies et petit à petit métabolisées. Comme pour ce qui concerne la maturation de l'être, le symbole prend du temps et du silence plein.

Et c'est de là que s'accomplit le sentiment d'incomplétude. Le rite n'est ici ni affiché, ni discouru, il se tient dans la pratique, dans la création et, en ce sens, il est éloquent du grand silence vers quoi va ce malade. Il fait large part au doute qui étreint, à l'angoissement qui parfois submerge. Il fait y entrer. Il est possible qu'au bout du compte, oui, être entré console plus que d'avoir esquivé.

Ce rite d'avant la mort a sans doute toujours existé. On ne sait. En revanche, ce qui amplifierait sa présence aujourd'hui serait de deux ordres. En premier lieu, la chronicisation de la maladie vient bouleverser nos conceptions du temps et ses usages: en effet, la limitation fonctionnelle provoque une identification personnelle sous des registres parfois jusque-là méconnus, sinon méprisés, faisant se reposer dans l'intimité des êtres cette question: s'il n'y a plus de continent à explorer, comment manœuvrer avec une limitation physique, ou autre, tout en ne s'identifiant pas seulement comme personne malade? En d'autres termes, dans le métissage que propose notre culture, y aurait-il place à découvrir les territoires d'une nouvelle interdépendance, une vision distanciée du monde, autorisée par la maladie? Le rite pourrait bien contribuer à cette quête en ceci qu'il fait se relier l'individu à ce qui le déborde, l'intemporalité, l'environnement, si ce n'est le cosmos. Il fait se décrisper du moi. En second lieu, si les individus et les cultures savent plus ou moins confusément comment le rite aide à vivre, il se peut bien que nos contemporains cherchent une alternative à la déroute actuelle des rites funéraires et réparent par défense anticipatoire le vacuum alors créé. On peut même se demander si le rite avant, mis en place par ses premiers acteurs, ne stimulerait pas la mise en scène rituelle de l'après, par leurs survivants.

Mais avançons encore sur la piste de la niche écologique, dans la pédagogie du symbole hors technique. Comme nous venons de le voir au plan microscopique, le rite fait prendre acte de la présence de la mort pour un être et ses proches. Ces derniers admettent la réalité du tout autre, du grand changement, de l'altérité. Bien mieux, ils mûrissent au lieu de simplement se justifier. Par recadrage, peut-on déplacer ce même rite, au plan macroscopique?

\section{DU RITE SALUT AU CHANGEMENT DES$$
\text { REPRÉSENTATIONS DE LA MORT }
$$

Car ce qui change, ce n'est pas seulement un être qui entre comme un dit vivant dans sa mort, c'est l'ordre même des conceptions de la mort. Si le rite fait composer avec le changement, et fait transformer, peut-on également interpréter son existence comme une réponse au désarroi provoqué par les transformations actuelles du statut de la mort? Prenons trois de ces nouvelles représentations.

$1^{\text {re }}$ représentation: la mort comme résolution d'un problème avec la vie. Il arrive en effet que la mort signifie la délivrance et délivrance de tant de souffrances à la fois muettes et hurlantes sous la douleur: de conflits mortifères au sein de la vie organisationnelle, relationnelle, intrapsychique, de frustrations ou d'échecs; de tristesses non entendues, toujours exprimées d'une

\section{LE RITE FAIT PRENDRE ACTE}

\section{DE LA PRÉSENCE DE LA MORT}

\section{POUR UN ÊTRE ET SES PROCHES.}

CES DERNIERS ADMETTENT

\section{LA RÉALITÉ DU TOUT AUTRE,}

DU GRAND CHANGEMENT,

\section{DE L'ALTÉRITÉ.}

manière ou d'une autre; de contradictions non travaillées par sentiment d'impuissance... Parfois même la mort ou plutôt ce qui y conduit est accepté dans une secrète volupté, parce qu'enfin, les événements de la vie, ou encore le destin, se chargent de faire aboutir ce qui pour d'aucuns n'a pu être résolu par l'acte suicidaire, en dépit de ses fantaisies. En d'autres termes, pour beaucoup de nos contemporains, la mort arrive à point nommé. Elle délivre du mal de vivre chronique, inaperçu, agissant en bas bruit. Elle devient une bonne affaire. À ce chapitre elle peut également se redoubler d'une abdication. En effet, ne pouvant plus s'identifier à son statut d'avant la maladie, trouvant désormais dans le statut socialement imparti à la maladie une large part d'ambiguïtés non avouées, éprouvant confusément une difficulté de recul devant les impasses culturelles et économiques enkystées dans l'institution médico-hospitalière, le malade se trouve saturé de non-sens. Cet embarras de soi peut bien se concentrer sous le fameux «je ne veux pas déranger»: le temps des autres. La demande sociale d'euthanasie serait de la sorte tapissée de cet engorgement de l'affect, de cette non-désirabilité sociale. La bonne mort acquiesce à cette loi du marché et à une nouvelle civilité, tandis que la mauvaise signe le manque de talent au lâcher prise. Prise de quoi?

$2^{\mathrm{e}}$ représentation: la mort comme rejet du corps. On a dit et redit ceci à propos du corps contemporain: sa rutilante efficience comme outil de travail; la correspondance aux canons des modes comme moyen de séduction; la scarification, le tatouage comme indicateurs identitaires. Le corps est désormais à la personnalité ce que le fric est aux échanges marchands mondialisés. Alors habitant un corps dont on ne contrôle plus les humeurs, pas plus que les mouvements, devant et dans ce corps en dysfonctionnement, voire en déliquescence, que peut souhaiter le grand malade? Pointe l'immense paradoxe du faire semblant que ce corps n'existe pas, sauf pour le langage du mal, qui, lui, nous gratifie dans nos interventions. Du côté des patients, la réclamation sur la douleur, mine de rien, ramasse une boule d'indicible et d'irrecevable. Et se redouble l'embarras de soi, d'abord on l'a vu, traînant mal le non-amour de la vie, puis horrifié de cet habitat-corps qui abandonne et dont on voudrait vite se débarrasser. C'est paradoxalement le corps qui prend toute la place, qui obnubile, qui pollue. L'esthétisme d'une technologie soft trouve alors sa niche. Mais c'est mieux, tout de même mieux que les odeurs mélangées de mauvaise cuisine et d'urine qui coupent encore plus du monde ces vieillards aux yeux vides, affaissés, murmurant pour euxmêmes d'étranges mélopées.

$3^{\text {e }}$ changement: la mort comme déni de la violence. On pense évidemment à la violence intrinsèque de toute mort, à sa dureté, à ce qu'elle inflige à toute détermination humaine. Cette violence-là est obscène et va du coup se liquider ailleurs dans notre culture, notamment dans les conduites à risque, et puis dans le culte du morbide ${ }^{3}$. On pense aussi à la violence intrusive des technologies hard, d'ailleurs pas forcément impertinentes. Mais ce qui est nouveau, c'est ce qui peut faire violence au malade et au mourant en dépit et même avec nos bonnes intentions. Car ce qui ne se dit pas, mais qui suinte néanmoins, c'est la violence tranquille des injonctions, souvent paradoxales, parfois incantatoires, sur l'acceptation et la pacification. On trouve là un des effets pervers des 
modèles de mourir: ces effets désignent l'acceptation comme l'ultime performance. Comme quoi la culture imbibe, même à notre corps défendant. Le temps étant ici compté, beaucoup de proches perçoivent l'avant-mort comme une course à obstacles, les yeux rivés sur le moment où le malade acquiescera enfin à son sort et livrera son ultime message. Se découvre sous ce compte à rebours une obsession de contrôle de la mort: sous support technique, notamment par médecine interposée, puis par «trucologie» relationnelle (on me pardonnera ce néologisme), mais surtout par libre arbitre individuel comme automatisme de prévision. Coiffant le tout, la perfusion de la douceur vient édulcorer cette violence. (Bien sûr, ici aussi, je pousse le trait: on comprendra qu'il ne s'agit là pas tant d'un reflet exhaustif que d'un contrepoint à l'idéologie dominante.)

En somme, résolution d'un problème avec la vie, rejet d'un corps inopérant, déni de la violence, ces trois changements dans l'histoire des mentalités ont sans doute contribué à amplifier la souffrance de qui s'approche de la mort. Il se peut bien que, en plus de composer avec son destin et son histoire, le grand malade se ressente écrasé dans le jeu des valeurs culturelles qui le rendent indésirable. Il se peut bien alors que le passage dans la mort procède d'un gigantesque euphémisme et d'une entreprise de mise au tabou de vérités insoutenables.

\section{LE PASSAGE DE LA BONNE À LA BELLE MORT: LA VOLONTÉ DE CONTRÔLE RÉEL DU TEMPS}

Car les valeurs se dévoilent bien, pour peu qu'on tire sur le voile. Du passage de bonne à belle mort, il n'y a pas de hasard: la charité chrétienne n'est plus tant la valeur cardinale, talonnée de près par l'injonction pour l'individu de ne pas déchoir à ses propres yeux et, surtout, aux yeux des autres.

Ce qui est aussi visible, c'est le rapport au temps : la bonne mort arrive à son heure, la belle mort arrive quand je l'ai invitée, quand je suis prêt...

Le rapport à la conscience et aux autres, ces grands marqueurs de l'humanisme, se modifient aussi dans le passage de bonne à belle. La bonne mort la regarde venir comme un "écureuil dans la main» (un patient); de son côté, la belle est souhaitée dans l'hédonisme, sinon dans l'extase ${ }^{4}$. Ou dans l'instant. La bonne mort est concernée par la mise au clair de ses relations, fussent-elles décimantes, la belle mort ménage le souvenir pour l'après, logique de calcul affectif pesant.

Si la mort ne venait pas accuser le rapport à la vie, au corps et à la violence, les humains ne se désâmeraient pas tant à en prescrire les contours. L'histoire des mentalités ne se serait pas tant attardée sur les modèles de mort.

Au bilan pour cet aujourd'hui, bien sûr partiel, nous ne voulons pas tant être consolés que rassurés; nous voulons être protégés contre certaines vérités qui nous effraient, nous estimant faibles, désarmés, ou trop seuls. Justement, face à cette délivrance, ce rejet du corps, ce déni de la violence. C'est ainsi que les tabous gonflent et que nous acquiesçons à la logique du court terme.

Pour être consolés, encore aurait-il fallu sortir de la compulsivité à être rassurés, à se justifier. Encore nous faut-il avoir avoué la perte, le désarroi, l'indignation, la peine. Ici et désormais le rite ne bute pas que sur de l'existentiel pur ou encore des encoches relationnelles plus ou moins dramatiques. Il bute sur un rapport à la fin de la vie où se condensent les refus de vie et de mort de toute une vie, refus soigneusement entretenus par une culture qui prend entre autres sa technologie pour une suprême lanterne.

\section{FAIRE LA DIFFÉRENCE}

Il est autrement plus complexe de penser ce qui de la vie se déverse dans le mourir. Et là, en conclusion, de jouer les gradients entre deux pôles, celui de l'indifférenciation et celui du différentialisme. L'indifférenciation renverrait à l'application systématique et aisée des conceptions de bonne mort - ou de lutte contre la «male mort». Elle colmate bien les contradictions accumulées et tue symboliquement, puisqu'elle abolit la singularité. Le différentialisme, quant à lui, tiendrait dans la sacralisation de la singularité ou du culte de soi comme une île. En tension entre ces deux extrêmes, j'en appelle à un travail d'éducation populaire et évidemment d'éducation des malades: ce travail ne tient pour acquis aucun contenu des modèles. Il questionne ce qui de la puissance normalisante s'y déverse et ce qui se joue en termes de domination des consciences et de domestication bâclée de la violence de la mort. Ce travail s'ancre dans la construction symbolique de ce à quoi on veut rester relié par-delà la mort et, forcément, ce de quoi on se déleste. Bien mourir, ce serait alors passer dans l'ailleurs au plus près du désir qu'on en a, oui, mais avec le juste soupçon que, en mourant, nous réalisons une loi qui à la fois nous relie au temps des autres humains et nous dépasse. Car il arrive que l'on puisse être davantage consolés qu'effrayés - et donc, rassurés - par l'étendue de la vie qui ne se ramène pas à soi. L'accomplissement peut se réaliser dans l'incomplétude que vient aussi révéler toute mort. Accomplissement et incomplétude salués par le rite et par lui rencontrés.

\section{Bibliographie}

DES AULNIERS, Luce (2003). «Les invasions barbares, le contentement, l'angoisse, l'émoi », PRISME, no 41, p. 131-135.

DESAULNIERS, Luce (1997 [1989]). Itinérances de la maladie grave, Le temps des nomades, Paris, L'Harmattan, 624 p.

LECLERC, Rachel (2000). Noces de sable, Montréal, Boréal, 219 p.

M'UZAN, Michel de (1977). De l'art à la mort: itinéraire psychanalytique, Paris, Gallimard, 202 p.

SOLNIT, Rebecca (2002). L'art de marcher, trad. de l'américain par Oristelle BONIS, coll. Babel, Paris, Actes Sud, 395 p.

THOMAS, Louis-Vincent (1991). La mort en question. Traces de mort, mort des traces, coll. Nouvelles études anthropologiques, Paris, L'Harmattan, $536 \mathrm{p}$.

\section{Notes}

1. Le propos de cet article prend source dans mes travaux de recherche (notamment Itinérances de la maladie grave, Le temps des nomades, 1997). À partir de douze récits ethnographiques, cet ouvrage élabore une théorie sur les pratiques en situation de menace et en dégage à la fois une analyse critique du statut social dominant et un inventaire des fonctions du rite avant la mort, auxquels viennent se greffer depuis 1976 une pratique de supervision en milieu de santé et d'enseignement (particulièrement au sein $d u$ champ des études supérieures interdisciplinaires sur la mort, que j'ai fondé à l'Université du Québec à Montréal en 1980) et, enfin, comme anthropologue clinicienne. La plupart des pistes d'analyse ici proposées l'ont également été dans le cadre d'une conférence (inédite) tenue lors des Quinzièmes Entretiens du Centre Jacques-Cartier, à Lyon, en 2002, au sein du Colloque "Bien mourir», sous le titre «Le rite en contexte de technologisation, survivances, nouveautés et liens avec une belle mort».

2. Si je parle de tension "délicieuse », c'est en prenant distance de l'acception courante de «tension» qui la donne comme automatiquement souffrante et conflictuelle. C'est que la mise en tension, au sens d'équilibrage continu entre forces différentes, mais non nécessairement antagonistes, est bien constitutive des rapports entre vivants et des rapports entre la vie et la mort. Du coup, la dite tension peut être appréciée comme stimulante et source de joie, et de la sorte nous entraîner à concevoir de manière non évitante la présence des manifestations diverses de l'altérité.

3. Ce déni de la violence de la mort est forcément imparti au déni de la réalité de la mort; comme tout ce qui est dénié, cela finit par rebondir. Un ouvrage sur le statut ambigu de la mort, singulièrement à travers la fascination, est en cours d'édition.

4. On en tiendra pour exemple de valeur internationale le succès du film Les invasions barbares, de Denys Arcand, en 2003, auquel, parmi tant d'autres, j'ai consacré quelques lignes (voir Des Aulniers, 2003). 